\title{
Die Rezeption des Besitzbegriffs Jherings im niederländischen Recht
}

\section{Einleitung}

Die scheinbare Leichtigkeit, mit der der Besitzbegriff Jherings Eingang in der niederländischen Lehre fand, steht in krassem Widerspruch zu der überwiegend ablehnenden Haltung in der deutschen Literatur. Während das deutsche Recht an dem Besitzbegriff Savignys und damit an der Relevanz eines Besitzwillens festgehalten hat, entwickelte sich das niederländische Recht allmählich in Richtung des Besitzbegriffs Jherings. Mit dem Inkrafttreten des neuen niederländischen Bürgerlichen Gesetzbuchs (Burgerlijk Wetboek; BW) im Jahre 1992 ist diese Entwicklung sogar gesetzlich verankert worden.

In diesem Beitrag wird zunächst beschrieben, wie der Besitzbegriff Jherings unter der Geltung des alten niederländischen Bürgerlichen Gesetzbuch in der niederländischen Doktrin Eingang gefunden hat. Anschließend wird der Einfluss Jherings auf das Besitzrecht im neuen Bürgerlichen Gesetzbuch untersucht. Abschließend wird der niederländische Besitzbegriff mit dem deutschen Besitzbegriff verglichen, wodurch die Unterschiede zwischen dem Besitzbegriff Savignys und dem Jherings klar hervortreten. Vorangehend wird kurz der Kern des Besitzbegriffs Jherings skizziert.

Weil sich der niederländische und der deutsche Besitzbegriff in terminologischer Hinsicht voneinander unterscheiden, wird in diesem Beitrag von der im gemeinen Recht gängigen Terminologie ausgegangen. Derjenige, der die tatsächliche Herrschaft über die Sache ausübt, wird als Inhaber (im BW: houder; im BGB: Besitzer) bezeichnet. Derjenige, der die Sache für einen anderen innehat (im BW: houder voor een ander oder detentor; im BGB: Fremdbesitzer) wird als Detentor definiert. Derjenige, der die Sache für sich selbst innehat, wird als Besitzer (im BW: bezitter; im BGB: Eigenbesitzer) bezeichnet.

\section{Der Besitzbegriff Jherings}

Bekanntlich entwickelte Jhering in Der Besitzwille (1889) eine Gegenauffassung zum derzeit gängigen Besitzbegriff, der namentlich durch Savignys Recht des Besitzes geprägt wurde. Kern dieses Besitzbegriffs Savignys ist der Gedanke, dass sich der Besitz aus zwei 


\section{E.F. VERHEUL}

Elementen zusammensetzt: corpus und animus. ${ }^{1}$ Während corpus in dieser Besitzdefinition als die tatsächliche Herrschaft über die Sache verstanden wird, bezeichnet animus den Willen, die tatsächliche Gewalt für sich selbst und wie ein Eigentümer auszuüben (der sogenannte animus domini). Fehlt ein solcher animus domini, ist derjenige, der die tatsächliche Herrschaft ausübt, nicht Besitzer, sondern lediglich Detentor. Ein Detentor hat zwar die tatsächliche Herrschaft über die Sache, übt diese jedoch nicht für sich selbst, sondern für einen anderen aus: Er hat keinen animus domini, sondern einen animus alieno nomine possidendi. ${ }^{2}$

Jhering kritisierte diese Besitzdefinition, die er als Subjektivitätstheorie bezeichnet, ${ }^{3}$ da die subjektive Willensrichtung desjenigen, der die Sache innehat, für die Frage, ob Besitz oder Detention eintritt, maßgeblich sei. Für Jhering war problematisch, dass ein solcher animus domini außerordentlich schwer zu beweisen ist. ${ }^{4}$ Darüber hinaus hat die Subjektivitätstheorie zur Folge, dass die Entstehung und Fortdauer mittelbaren Besitzes völlig vom Willen des Detentors abhängig wird. Infolgedessen konnten die Zweckmäßigkeitserwägungen, die nach Jhering dem Unterschied zwischen Besitz und Detention zugrunde liegen, einfach dadurch untergraben werden, indem der Detentor seinen Willen ändert und den mittelbaren Besitz zu Fall bringt. ${ }^{5}$

Nach Jhering ist für die Beantwortung der Frage, ob der Inhaber einer Sache Detentor oder Besitzer ist, nicht maßgeblich, ob der Inhaber einen animus domini hat. Entscheidend für das Vorliegen von Detention oder Besitz ist nach Jhering nur der Rechtsgrund, kraft dessen der Inhaber die tatsächliche Herrschaft ausübt (causa detentionis). In dieser Theorie Jherings ist Besitz der normalerweise gegebene Ausgangspunt, während Detention nur ausnahmsweise eintritt, wenn das Recht aus Zweckmäßigkeitserwägungen jemandem den Besitz abspricht und deswegen Detention eintreten lässt. ${ }^{6}$ Diese Zweckmäßigkeitserwägungen sind das Interesse eines anderen: "Dem Detentor wird der Besitz abgesprochen, nicht aus einem in seiner eigenen Person gelegenen Grunde: weil es ihm an dem richtigen Besitzwillen fehlt, sondern im Interesse des Besitzherrn, um diesem die Rechtsstellung des Besitzers zuzuwenden." ${ }^{7}$ Der Detentor ist nach Jhering nicht deswegen kein Besitzer, weil es ihm an einem besonderen Besitzwillen fehlt, sondern weil das Recht ihm eben den Besitz

1 Statt vieler Savigny, Recht des Besitzes, 7. Auflage (1865), 108 ff., Puchta, Pandekten, 12. Auflage (1877), 184, Randa, Der Besitz, 3. Auflage (1879), 2 f., 12 und Windscheid-Kipp, Lehrbuch des Pandektenrechts, 9. Auflage (1906), 760.

2 Sintenis, Das practische gemeine Civilrecht (1844), 443 ff., Savigny (oben Fußnote 1), 109, Randa (oben Fußnote 1), 19 ff., Brinz, Lehrbuch der Pandekten. Band I, 2. Auflage (1873), 495, Puchta (oben Fußnote 1), 184 und Arndts, Lehrbuch der Pandekten, 9. Auflage (1877), 206 f. Vgl. auch Windscheid-Kipp (oben Fußnote 1), 772.

3 Jhering, Der Besitzwille (1889), $7 \mathrm{f}$.

4 Zum Beweisproblem prägnant Jhering (oben Fußnote 3), 162 ff.

$5 \quad$ Siehe z. B. Jhering (oben Fußnote 3), 13 f, 173.

$6 \quad$ Jhering (oben Fußnote 3), 8, 17, 76 ff., 201, 323, 364 ff.

7 Jhering (oben Fußnote 3), 65. 


\section{DIE REZEPTION DES BESITZBEGRIFFS JHERINGS}

abspricht, da er in einem Rechtsverhältnis mit einem anderen steht, dem der Besitz zugesprochen wird. ${ }^{8}$

Die Unterschiede zwischen den beiden Theorien lassen sich prägnant folgendermaßen wiedergeben. ${ }^{9}$ Während nach der Subjektivitätstheorie Detention mit einer hinzukommenden, besonderen Willensrichtung zu Besitz führt, hat in der Theorie Jherings jede tatsächliche Herrschaft Besitz zu Folge, sofern nicht etwas (ein Rechtsverhältnis) gegeben ist, das dem Inhaber den Besitz abspricht, infolgedessen er nur Detentor ist. Bei Jhering ist Besitz also der Regelfall und Detention die Ausnahme, während in der Subjektivitätstheorie hingegen Detention den Ausgangspunkt bildet und nur ein besonderer Wille diese Detention in Besitz umwandelt.

\section{Die Beweisbarkeit des animus domini}

Jhering hielt die Subjektivitätstheorie nicht nur theoretisch für verfehlt, sondern auch für gänzlich unpraktisch, weil es unmöglich sei, den Beweis dieser subjektiven Willensrichtung zu erbringen. Der Besitzschutz gerate damit zur Farce, weil derjenige, der den Besitzschutz beanspruchen würde, den Beweis eines animus domini liefern müsse. Gerade im Rahmen der Beweisbarkeit dieses Besitzwillens findet der Besitzbegriff Jherings zum ersten Mal im niederländischen Recht ernsthaft Erwähnung, und zwar anlässlich eines Urteils des Hoge Raad. ${ }^{10}$ Der Fall war folgendermaßen. Ein Fuhrmann wurde in seinem Besitz gestört und klagte auf Beseitigung dieser Störung. Er bewies, dass er das betreffende Grundstück schon länger als ein Jahr besaß und dass der Beklagte ihn im Besitz störte. Man sollte also annehmen, dass der Klage stattgegeben werden würde. Trotzdem wird die Klage von dem Gericht erster Instanz abgewiesen und die dagegen gerichtete Revision wurde als unbegründet zurückgewiesen. Der Hoge Raad begründet sein Urteil damit, dass der Kläger nur dargelegt hat, dass er das Grundstück besitzt, und nicht, dass er das Grundstück auch wie ein Eigentümer besessen hat, sodass die Klage zurückgewiesen werden muss, weil die Klage wegen Besitzstörung nur demjenigen offensteht, der die Sache wie ein Eigentümer besitzt.

Es ist gerade diese Argumentation, die Jhering für verfehlt hielt und die Van Oven in einer Anmerkung zu dem Urteil nach dem Vorbild Jherings kritisiert. ${ }^{11}$ Van Oven zufolge geht der Hoge Raad von einer verfehlten Interpretation des niederländischen Besitzbegriffs aus, indem er sich "der alten und verwerflichen Lehre ergibt, die meint, dass der animus einen selbstständigen Teil des Besitzbegriffs bildet". ${ }^{12}$ Stattdessen hätte er nach Van Oven

Jhering (oben Fußnote 3), 64.

Siehe schematisch Jhering (oben Fußnote 3), 52 ff.

HR, Urt. v. 12.06.1908, W. 8722.

Van Oven, WPNR 2027 (1908), 549 ff.

Van Oven WPNR 2027 (1908), 549 (550). Siehe bereits kritisch Van Oven, De bezitsbescherming en hare functies (1905), $177 \mathrm{ff}$. 


\section{E.F. VERHEUL}

den Besitzbegriff Jherings zum Ausgangspunkt nehmen müssen. Auf den ersten Blick könnte man jedoch meinen, dass das Urteil an sich nicht die Unrichtigkeit der animus-Lehre belegt, sondern nur verkennt, dass Artikel 590 BW (a. F.) dem Besitzer gerade in diesem Punkt zu Hilfe kommt. ${ }^{13}$ Entsprechend diesem Artikel wird vermutet, dass derjenige, der die Sache besitzt, sie wie ein Eigentümer besitzt. Meijers stellt im Jahre 1921 fest, dass die Rechtsprechung des Hoge Raad zu diesem Artikel ein großes Rätsel ist. ${ }^{14}$ Der Auslegung des Hoge Raad zufolge erscheint die Vermutung erst dann anwendbar, wenn der Kläger den Beweis erbracht hat, dass er die Sache innehat und dass er die Sache auch wie ein Eigentümer innehatte. Damit reduziert er die Bedeutung des Artikels zu der banalen Feststellung, dass der Beklagte den Gegenbeweis dafür zu erbringen hat. ${ }^{15}$ Meijers wandte sich unter Bezug auf Jhering gegen diese Interpretation und legte dar, dass der Artikel gerade dazu dient, den schlichten Beweis der tatsächlichen Herrschaft ausreichen zu lassen, indem das Vorhandensein des Willens, die Sache wie ein Eigentümer innezuhaben, vermutet wird. ${ }^{16}$ Ab dem Jahre 1924 wird diese Auslegung von Artikel 590 BW (a. F.) tatsächlich vom Hoge Raad übernommen. ${ }^{17}$

Dem Anschein nach könnte man daraus jedoch folgern, dass sich der niederländische Besitzbegriff durchaus aus zwei Elementen zusammensetzt, und zwar aus der tatsächlichen Herrschaft über die Sache (Innehabung; corpus) und dem animus domini, sei es nur, dass die Existenz dieses Willens durch Artikel 590 BW (a. F.) vermutet wird. Jhering legte bereits dar, dass der Gesetzgeber die animus-Lehre mittels einer solchen Vermutung "gänzlich ungefährlich" macht. ${ }^{18}$ Ihm zufolge handelt es sich dabei in Wirklichkeit jedoch um eine halbe, künstliche Lösung und ist der Rückgriff auf eine solche Vermutung in Wirklichkeit eine Verabschiedung des animus-Erfordernisses. ${ }^{19}$ Auf vergleichbare Weise merkte Scholten an, dass die richtige Auslegung des Artikels 590 BW (a.F.) gerade "die Spitze von der bedenklichen Lehre des animus domini abgebrochen hat." 20 In der Tat werden mit einer solchen Vermutung unterm Strich die Ergebnisse des Besitzbegriffs Jherings erreicht: Besitz ist der Ausgangspunt und Detention muss bewiesen werden. Es verwundert deshalb nicht, dass Van Oven die Vermutung gerade als Ausdruck des Besitzbegriffs Jherings betrachtet, weil die Vermutung des Artikels 590 BW (a.F.) nicht dadurch widerlegt werden kann, dass der Beklagte beweist, dass der Kläger keinen animus domini hat, sondern nur durch die Tatsache, dass der Kläger, angefangen hat, für einen anderen zu besitzen. Entscheidend sei nach Van Oven also nicht, dass der Kläger nicht den Willen hat, die Sache wie ein Eigentümer innezuhaben, sondern nur, ob er die Sache aufgrund eines

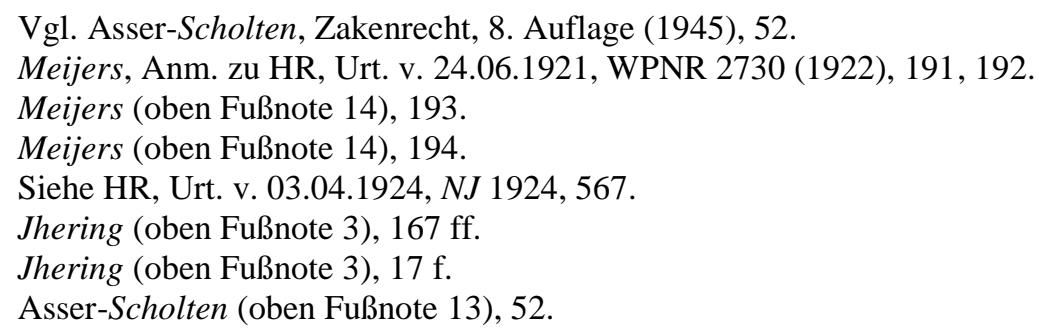




\section{DIE REZEPTION DES BESITZBEGRIFFS JHERINGS}

Rechtsverhältnisses für einen anderen innehat. ${ }^{21}$ Nicht das Fehlen des animus domini, sondern nur das Bestehen eines Rechtsverhältnisses, kraft dessen er die Sache für einen anderen innehat, könne die Vermutung beiseiteschieben. Hierin klingt deutlich der Ansatz Jherings durch, wonach nicht der Wille, sondern das Rechtsverhältnis darüber entscheidet, ob Besitz oder Detention vorliegt.

\section{Die weitere Rezeption des Besitzbegriffs Jherings}

Die prozessualen Schwierigkeiten bei der Feststellung von Besitz scheinen den Auftakt für eine breitere Akzeptanz des Besitzbegriffs Jherings in der niederländischen Doktrin zu bilden. Im Jahre 1913 erscheint die Dissertation von Van Kan, in der er umfassend überprüft, anhand welches Kriteriums beurteilt werden muss, ob Besitz oder Detention vorliegt. ${ }^{22}$ Obschon er in diesem Zusammenhang der Ansicht Jherings entgegentritt, ${ }^{23}$ gelangt er letztendlich zu Schlussfolgerungen, in denen die Ansicht Jherings ganz klar durchklingt. ${ }^{24}$ Auch Van Kan zufolge ist Besitz der Ausgangspunkt und Detention die Ausnahme. Er wendet sich gegen

\footnotetext{
"de fundamenteele onjuistheid van de gangbare voorstelling, die in de detentie het uitgangspunt en den grondslag ziet van de leer van het bezit. De detentie schraagt niet het Bezitsbegrip maar integendeel is het Bezitsbegrip primordiaal, de detentie het afgeleide begrip (...). De detentie is niet een rechtstreeksch product uit de ruimte-betrekking. Zij ontstaat eerst door een verkeersbeweging van den Bezitter."25
}

Van Kan folgert daher, dass es keinen prinzipiellen Unterschied zwischen Detention und Besitz gibt; ihm zufolge sind alle Besitzverhältnisse gleichartig, sodass grundsätzlich sowohl der Besitzer als auch der Detentor den Besitzschutz in Anspruch nehmen können müssen. Nur aus Zweckmäßigkeitserwägungen (die Bedürfnisse des Rechtsverkehrs) werde jemandem gelegentlich der Besitzschutz abgesprochen. ${ }^{26}$

Von wesentlicher Bedeutung ist die einflussreiche Bearbeitung des Asser-Kommentars, in dem Scholten sich dem Besitzbegriff Jherings unumwunden anschließt. ${ }^{27}$ Scholten zufolge hat Jhering zu Recht darauf hingewiesen, dass der animus domini bei der Unterscheidung zwischen Besitz und Detention keine Rolle spielt. Wenn verschiedene Personen

21 Van Oven (oben Fußnote 11), 551. Vgl. auch Van Oven, WPNR 2292 (1913), 577, 594, wo er spricht von Art. 590 BW (a.F.) als eine Vermutung die keine Vermutung ist, sondern eine reine Rechtsregel (vermoeden-dat-geen-vermoeden-is-maar-een-zuivere-rechtsregel).

22 Van Kan, Bezitsverhoudingen (1913).

23 Van Kan (oben Fußnote 22), 46 ff.

24 Siehe z. B. Van Kan (oben Fußnote 22), 104 ff.

25 Van Kan (oben Fußnote 22), 101 und 103.

26 Siehe bes. Van Kan (oben Fußnote 22), 104 ff.

27 Asser-Scholten (oben Fußnote 13), 48 ff. 


\section{E.F. VERHEUL}

Herrschaft über eine Sache haben und diese Personen in einem Verhältnis zueinander stehen, so argumentiert Scholten, habe das Recht zu entscheiden, wer Besitzer der Sache ist. ${ }^{28}$ Dies geschieht nach Scholten nur aufgrund rechtlicher Gründe: Weil diese Personen in einem Rechtsverhältnis zueinander stehen, werde einer von ihnen aufgrund dieses Rechtsverhältnisses der Besitz zugesprochen und die andere als Detentor bezeichnet. Diese Qualifikation sei eine objektive, sodass der Wille dieser Personen bedeutungslos sei. ${ }^{29}$ Wäre dies anders, dann könne der Mieter einfach dadurch zum Besitzer werden, indem er sich fortan wie ein Eigentümer verhalten würde. ${ }^{30}$ Das würde jedoch die dem Rechtsverhältnis zugrunde liegenden Zweckmäßigkeitserwägungen durchkreuzen. Umgekehrt würde derjenige, der eine Sache zu Unrecht als einem anderen gehörend ansehe, nicht Besitzer sein, sondern lediglich Detentor. Scholten zufolge ist das nicht haltbar: Das Rechtsverhältnis entscheide zwischen Besitz und Detention; fehle ein solches Rechtsverhältnis, sei derjenige, der die tatsächliche Macht ausübt ohne Weiteres Besitzer. ${ }^{31}$ Seine Schlussfolgerung entspricht daher auch ganz klar der Auffassung Jherings:

\footnotetext{
"Wij kunnen dat alles ook zoo samenvatten: bezit is niet detentie met een bijzondere gezindheid, den wil om eigenaar te zijn, maar detentie is bezit dat niet als zoodanig wordt erkend, omdat een bijzondere rechtsverhouding bewezen is." 32
}

Diese zunehmende Betonung des Rechtsverhältnisses statt des Willens als entscheidendes Kriterium für die Feststellung von Besitz oder Detention, hatte nicht nur theoretische Bedeutung für den Besitzbegriff, sondern bildete auch die Grundlage für die Entwicklung neuer Rechtsfiguren.

So bahnte die neue Definition des Besitzbegriffs den Weg für die Übereignung von Sachen, über die ein Dritter für den Veräußerer die tatsächliche Macht ausübt, ohne Mitwirkung dieses Dritten. Während diese Übereignungsvariante, die heutzutage im niederländischen Recht als traditio longa manu bezeichnet wird, in der niederländischen Doktrin seit alters kaum zur Sprache kommt, weil man meinte, dass dafür erforderlich ist, dass der Dritte seinen Willen dadurch ändert, dass er die tatsächliche Gewalt fortan für den Erwerber ausüben wird, ${ }^{33}$ entscheidet der Hoge Raad im Jahre 1929, dass für eine solche Übereignung nicht erforderlich ist, dass der Dritte seinen Willen zugunsten des Erwerbers ändert, weil die Rolle des Dritten im Rahmen der Übereignung "völlig passiv" ist. ${ }^{34}$ Nach diesem Urteil wird diese Übereignungsart in der Literatur fast einhellig begrüßt und mit dem Argument

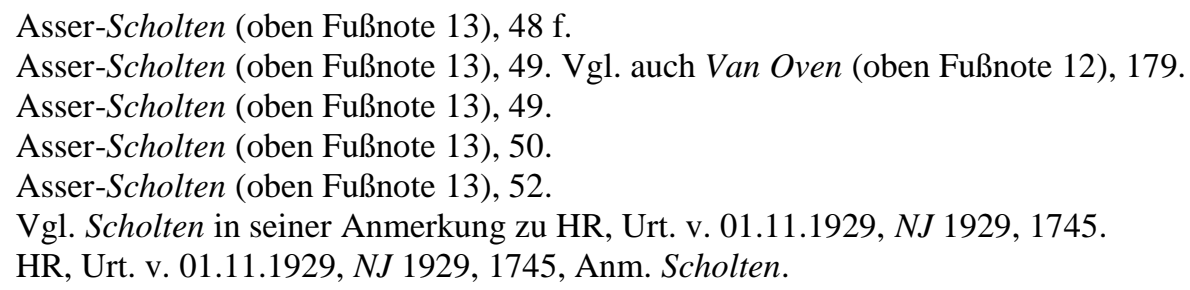




\section{DIE REZEPTION DES BESITZBEGRIFFS JHERINGS}

begründet, dass nicht der Wille des Dritten ausschlaggebend ist, sondern das Rechtsverhältnis, kraft dessen der Dritte die Sache innehat, sodass es möglich ist, die Sache ohne Mitwirkung des Dritten zu übereignen. ${ }^{35}$

Das Rechtsverhältnis als entscheidendes Kriterium wird auch bei der Übereignung künftiger Sachen und beim Eigentumserwerb bei mittelbarer Stellvertretung in Anspruch genommen. Ursprünglich überwog die Auffassung, dass der Eigentumserwerb in solchen Fällen vom tatsächlichen Willen desjenigen, der die künftige Sache antizipiert übereignet, oder desjenigen, der die Sache als Stellvertreter in Empfang nimmt, abhängt. Im Laufe der Zeit wird es jedoch immer mehr für möglich gehalten, dass das antizipierte Konstitut oder das Rechtsverhältnis zwischen Stellvertreter und Geschäftsherrn zu einer Bindung des Veräußerers bzw. Stellvertreters führt: Das Rechtsverhältnis zwischen demjenigen, der die künftige Sache antizipiert übereignet, und dem Erwerber bzw. zwischen dem Stellvertreter und dem Geschäftsherrn hat zur Folge, dass der Erwerber oder Geschäftsherr ohne Weiteres das Eigentum in dem Moment erwirbt, in dem der Veräußerer oder der Vertreter die tatsächliche Herrschaft über die Sache erwirbt. ${ }^{36}$ Unter der Geltung des alten niederländischen Gesetzbuchs blieb die Übereignung künftiger Sachen und der Eigentumserwerb bei mittelbarer Stellvertretung jedoch lange Zeit strittig. ${ }^{37}$ Erst im Ausklang dieses Gesetzbuchs wurden diese Figuren vom Hoge Raad akzeptiert, wobei er dem neuen niederländischen Bürgerlichen Gesetzbuch vorgriff, in dem das Rechtsverhältnis unumwunden als zentrales Kriterium verankert ist. ${ }^{38}$

\section{Die Eingliederung des neuen Besitzbegriffs in der damaligen gesetzlichen Terminologie}

Für ein richtiges Verständnis des heutigen niederländischen Besitzrechts ist es unerlässlich,

35 Vgl. Scholten in seiner Anmerkung (oben Fußnote 33) 1750, Eggens, Anm. zu HR, Urt. v. 01.11.1929, WPNR 3134 (1930), 32, 33, De Bruijn, WPNR 3153 (1930), 332, 334. Sehr kritisch hingegen Scheltema, Het stelsel van roerend goed in het B. W. van 1838 tot heden, Gedenkboek Burgerlijk Wetboek 18381938 (1938), 406, der sich noch ganz auf die animus-Lehre stützt: Der Hoge Raad verkenne, dass der mittelbare Besitz von dem Willen des Detentors abhängig ist. Früher auch so Meijers, Anm. zu HR, Urt. v. 16.03.1933, NJ 1933, 790, 792, der die Möglichkeit einer solchen Übereignung zwar akzeptierte, sie aber anhand eines unterstellten Willens des Detentors, die Sache auch für den Erwerber innehaben zu wollen, erklärte. In seinem Entwurf hat er dem Rechtsverhältnis hingegen entscheidende Bedeutung beigemessen. Siehe dazu Van der Lelij, Levering van roerende zaken door middel van een zakenrechtelijk waardepapier (1994), $47 \mathrm{ff}$.

36 Meijers, Eigendomsoverdracht tot zekerheid (1936), 252f., Wiarda, Overdracht van toekomstige rechten (1940), 38 ff., Pitlo, WPNR 3691 (1940), 409 ff., Asser-Scholten (oben Fußnote 13), 184 ff., Veenhoven, Eigendomsoverdracht tot zekerheid (1955), 52f. A.A. Langemeijer, NJB 10 (1935), 489, 494, Scheltema (oben Fußnote 35), 398 ff., Houwing, Overdracht van toekomstige rechten (1940), 22 ff., Gerbrandy, WPNR 1961 (4679), 245 ff.

37 Zum damaligen Streitstand ausführlich Van Swaaij, Beschikken en rechtsovergang (2000), $11 \mathrm{ff}$.

38 Siehe HR, Urt. v. 23.09.1994, NJ 1996, 461, Anm. Kleijn (zum Erwerb bei mittelbarer Stellvertretung) und HR, Urt. v. 24.03.1995, NJ 1996, 158, Anm. Kleijn (zur Übereignung künftiger Sachen). 


\section{E.F. VERHEUL}

sich zu vergegenwärtigen, dass die oben beschriebene Rezeption des Besitzbegriffs Jherings in einem System erfolgte, das auf den Grundlagen der animus-Lehre beruhte. So definierten die gesetzlichen Bestimmungen den Besitz als die Ausübung der tatsächlichen Macht über eine Sache "als ihm gehörend" (Art. 585 BW a.F.) oder "für sich selbst" (Art. 590 BW a.F.), während die Ausübung der tatsächlichen Herrschaft "für einen anderen" (Art. 591 BW a.F.) als Detention bezeichnet wurde. Die Rezeption des Besitzbegriffs Jherings und die Betonung des Rechtsverhältnisses als entscheidendes Kriterium mussten also mit diesen gesetzlichen Bestimmungen in Einklang gebracht worden.

Diese Harmonisierung wurde erreicht, indem die neue Lehre von "einem durch das Rechtsverhältnis gebundenen Willen" 39 sprach, sodass einerseits das animus-Erfordernis aufrechterhalten werden konnte, dieses Erfordernis anderersits jedoch anhand des Rechtsverhältnisses festgestellt wurde. Die Konstruktion des durch das Rechtsverhältnis gebundenen Willens ermöglichte also eine gewisse Überbrückung zwischen der gesetzlichen Besitzdefinition, in der die animus-Lehre durchklingt, und dem Besitzbegriff Jherings, indem an den herkömmlichen Erfordernissen (corpus und animus) festgehalten wurde, wenngleich die Frage, ob Besitz oder Detention vorliegt, letztendlich anhand des Rechtsverhältnisses beurteilt wurde. Nach dieser neueren Auffassung ermöglichte das Abschließen des Rechtsverhältnisses namentlich eine Willensbildung des Inhabers, sodass im Ergebnis nicht dessen Willen, sondern dem Rechtsverhältnis entscheidende Bedeutung beigemessen wurde. ${ }^{40}$ Mit diesem Ansatz kann die animus-Lehre als Fassade zwar aufrechterhalten werden, wird dem Willen in Wirklichkeit jedoch keine entscheidende Bedeutung beigemessen. Sobald man namentlich akzeptiert, dass das Rechtsverhältnis den Willen desjenigen, der die tatsächliche Herrschaft über die Sache ausübt, binden kann, wird in Wirklichkeit nicht auf den Willen, sondern nur auf das Rechtsverhältnis als entscheidendes Kriterium abgestellt. ${ }^{41}$

\section{Der Besitzbegriff im (neuen) niederländischen BW}

Diese Konstruktion des durch das Rechtsverhältnis gebundenen Willens, die den Besitzbegriff Jherings mit der derzeit gängigen gesetzlichen Terminologie in Einklang bracht, liegt auch dem heutigen niederländischen BW zugrunde. Mit der Neukodifikation des niederländischen Rechts wurde namentlich nicht ein neues bürgerliches Recht auf völlig anderer Grundlage beabsichtigt, sondern lediglich eine Modernisierung des geltenden

39 Siehe z.B. Pitlo, WPNR 3691 (1940), 409 ff. Vgl. auch Suijling, Inleiding tot het Burgerlijk Recht. V. Zakenrecht (1940), 114, der vom unterstellten Detentionswillen spricht.

40 Vgl. Zwalve, GrOM 6 (1989), 88, 116, der anmerkt, dass man so versuchte, die Bindung an das Rechtsverhältnis mit dem Festhalten an der animus-Lehre in Einklang zu bringen, damit aber zwei unversöhnliche Ausgangspunkte zu vereinen trachtete. Siehe auch Pitlo, WPNR 3691 (1940), 409, 411: "Of wij zeggen, dat de rechtsverhouding beslist, of dat wij zeggen dat de wil beslist, is in wezen hetzelfde. Want zoowel de rechtsverhouding als de wil zijn bepaald door het eigen behooren."

41 Jhering (oben Fußnote 3), 16. 


\section{DIE REZEPTION DES BESITZBEGRIFFS JHERINGS}

Rechts angestrebt, indem die Ergebnisse der Rechtsprechung und Dogmatik, die sich in Bezug auf den Wortlaut des Gesetzes mehr und mehr freier aufstellten, im neuen Gesetzbuch festgelegt wurden. ${ }^{42}$ Sofern möglich, wurde also am System des alten BW festgehalten. Das gilt auch für das neue Besitzrecht.

Aufgrund des zentralen Artikels des Besitzrechts (Art. 3:107 BW) ist derjenige Besitzer, der die Sache für sich selbst innehat, während ein Detentor die Sache für einen anderen innehat. Auf den ersten Blick scheint der niederländische Gesetzgeber mit dieser Definition von der animus-Lehre auszugehen, weil die Innehabung zum Ausgangspunkt genommen wird und anscheinend anhand des Willens des Inhabers entschieden werden muss, ob er Besitzer oder Detentor ist: Hat er die Sache für sich selbst inne, ist er Besitzer; übt er hingegen die tatsächliche Herrschaft für einen anderen aus, ist er Detentor.

Dieser Schein trügt allerdings. Obwohl das Gesetz sich der Begrifflichkeiten "für sich selbst" und "für einen anderen" bedient, wird nicht anhand des Kriteriums eines Besitzwillens beurteilt, ob jemand die Sache für sich selbst oder für einen anderen innehat. Artikel 3:108 BW verdeutlicht, dass anhand der Verkehrsauffassung unter Berücksichtigung der "nachstehenden gesetzlichen Bestimmungen" beurteilt werden muss, ob jemand die tatsächliche Herrschaft für sich selbst oder für einen anderen ausübt. Das neue Besitzrecht hat also zwar bei der Definition von Besitz und Detention an der alten Unterscheidung zwischen Innehabung "für sich selbst" und "für einen anderen" festgehalten, für die Antwort auf die Frage, ob Detention oder Besitz vorliegt, auf den Besitzwillen als entscheidendes Kriterium verzichtet.

Indessen wird von einigen Autoren behauptet, dass der Wille im Ergebnis durchaus entscheidend ist, mit der Maßgabe, dass es sich nicht um einen psychischen oder innerlichen Willen handelt, sondern um den nach außen erkennbaren Willen, weil die Verkehrsauffassung einen derartigen Willen berücksichtigen wird, sodass der Wille über die Verkehrsauffassung indirekt Relevanz hat. ${ }^{43}$ In diesem Zusammenhang wird auf eine Bemerkung in den Materialien des BW hingewiesen, wo angemerkt wird dass,

\footnotetext{
"de interne wil om als eigenaar op te treden, een animus domini, voor het zijn van bezitter van geen betekenis is. Het zijn uiterlijke feiten, waaraan in het verkeer een erkenning van bezit geknoopt wordt, die voor het zijn van bezitter beslissend zijn, al zijn dit feiten die in de regel op het bestaan van een animus domini wijzen." 44
}

Dieser Argumentation zufolge sind die nach außen erkennbaren Tatsachen entscheidend,

\footnotetext{
42 Siehe Van Zeben, Parlementaire Geschiedenis van het Nieuwe Burgerlijk Wetboek. Algemeen Deel (1962), $121 \mathrm{ff}$. Siehe ausführlich zu diesem Hintergrund der Neukodifikation Hartkamp, Aard en opzet van het nieuwe vermogensrecht (2017), $7 \mathrm{ff}$.

43 Beekhuis, WPNR 4461 (1956), 405 ff., Van der Lelij (oben Fußnote 35), 21 f., Rank-Berenschot, Bezit (2012), 19 ff., Snijders/Rank-Berenschot, Goederenrecht, 6. Auflage (2017), 99 f.

44 Van Zeben/Du Pon, Parlementaire Geschiedenis van het Nieuwe Burgerlijk Wetboek. Boek 3 (1981), 428.
} 


\section{E.F. VERHEUL}

sodass der Besitzwille aus der Art und Weise hergeleitet werden kann, in der der Inhaber die tatsächliche Herrschaft über die Sache ausübt. Dieser Ansatz ist jedoch aus zwei Gründen verfehlt. An erster Stelle lässt sich aus der Art und Weise, in der der Inhaber die tatsächliche Herrschaft ausübt, nicht ableiten, ob er einen Besitzwillen hat. ${ }^{45}$ Die nach außen erkennbaren Tatsachen und die Verkehrsauffassung sind mit anderen Worten nicht distinktiv (Abschnitt 6.1). Zudem wird zweitens die Verkehrsauffassung als entscheidendes Kriterium durch die nachstehenden gesetzlichen Bestimmungen beiseitegeschoben, sodass letztendlich das Rechtsverhältnis Vorrang vor den nach außen erkennbaren Tatsachen hat (Abschnitt 6.2).

\subsection{Die Beurteilung aufgrund der Verkehrsauffassung}

Der Gedanke, dass aus der Art und Weise, in der der Inhaber die tatsächliche Herrschaft über die Sache ausübt, abgeleitet werden kann, ob derjenige Besitzer oder Detentor ist, weil ein Besitzwille in dieser Ausübung der tatsächlichen Herrschaft zum Ausdruck kommt, ist untauglich, weil diese nach außen erkennbaren Tatsachen für die Feststellung von Besitz oder Detention keine auschlaggebenden Indizien bieten. ${ }^{46}$ Handlungen, die nur ein Besitzer vorzunehmen pflegt, sind eher selten. Ein solcher Ansatz wird jedoch von einigen Autoren befürwortet. So behauptet Reehuis, dass man aus dem Fahren auf einem Fahrrad oder dem Abstellen des Fahrrads in der Fahrradstation nicht ableiten kann, ob derjenige Besitzer des Fahrrads ist, weil diese Handlungen auch von einem Detentor vorgenommen werden können. ${ }^{47}$ Ähnliches gilt Reehuis zufolge zum Beispiel für denjenigen, der ein Namensschild an die Haustür stellt. Auch aus einer solchen Handlung kann nach Reehuis kein Besitz abgeleitet werden, weil ein Detentor entsprechende Handlungen auch vornimmt. Lediglich die Handlungen, die allein ein Eigentümer vorzunehmen pflegt, wie das Wechseln eines Fahrradreifens oder die Montage eines Heizkessels in einer Wohnung, würden auf Besitz hindeuten. ${ }^{48}$

Wie Scholten jedoch zu Recht bemerkt, gibt es viele Fälle, in denen sich das Verhalten eines Detentors in nichts vom Verhalten eines Eigentümers unterscheidet, sodass man "nur aus dem Rechtsverhältnis, nicht aus dem tatsächlichen Verhalten bezüglich der Sache ableiten kann, ob derjenige die Sache besitzt oder für einen anderen innehat." ${ }^{49}$ Wenn es eine solche Handlung, die nur ein Besitzer vorzunehmen pflegt, ausnahmsweise trotzdem gäbe, würde aus solchen Handlungen dennoch nicht ohne Weiteres Besitz abgleitet werden können, weil - wie unten noch ausführlicher dargestellt wird (Abschnitt 6.2) - der Detentor auch dann

\footnotetext{
$45 \quad$ Vgl. Van Oven, WPNR 4539 (1958), 241, 242.

46 Vgl. Struycken, De numerus clausus in het goederenrecht (2007), 291.

47 Pitlo-Reehuis/Heisterkamp, Goederenrecht, 13. Auflage (2012), 299.

48 Pitlo-Reehuis/Heisterkamp (oben Fußnote 47), 300.

49 Asser-Scholten (oben Fußnote 13), 49 f. Vgl. Jhering (oben Fußnote 3), 309 f. zur Detention des Pächters.
} 


\title{
DIE REZEPTION DES BESITZBEGRIFFS JHERINGS
}

Detentor bleibt, wenn er gerade diese Besitzhandlungen vornehmen würde. Auch wenn die nach außen erkennbaren Tatsachen in einem solchen Fall also auf Besitz hinzudeuten scheinen, ist in Wirklichkeit nur Detention gegeben. Letztendlich kann aus solchen Handlungen also nur Besitz abgeleitet werden, wenn kein Rechtsverhältnis vorliegt, aufgrund dessen Detention gegeben ist.

Zweifelhaft ist darüber hinaus, dass die Anzahl der Handlungen, die für die Feststellung von Besitz geeignet sind, stark eingeschränkt wird. Dadurch erhebt sich unter anderem die Frage, wie die tatsächliche Herrschaft qualifiziert werden muss, solange derjenige, der die tatsächliche Herrschaft über die Sache hat, noch keine für die Feststellung von Besitz relevante Handlungen ausgeführt hat. Ist derjenige noch kein Besitzer? Und sobald er dann hinterher eine entsprechende Besitzhandlung vornimmt, stellt sich die Frage, ob der Besitz erst dann erworben wird, oder als schon immer vorhanden anzusehen ist. ${ }^{50}$ Auf die Schwierigkeiten, die mit diesem Ansatz einhergehen, hat auch Jhering schon hingewiesen, und zwar im Rahmen der Frage, welche Besitzhandlungen (actus possessori) entscheidend sind:

\begin{abstract}
"Es figuriren darunter [unter den actus possessori; EFV] neben solchen die nur der Eigenthümer vornehmen darf (Aufbau, Niederreißung von Gebäuden, Fällung von Holz, Eintragung ins Kataster), auch solche, die auch dem Pächter zustehen (Bestellung des Ackers, Ernte, Austreiben des Viehes). Auf jene konnte man den Beweis unmöglich beschränken, denn damit wäre mit dem Pächter zugleich der wirkliche Eigenthümer, der bisher keine Veranlassung hatte sie vorzunehmen, ausgeschlossen worden; erklärte man aber diese für ausreichend, so schlüpfte mit dem Eigenthümer auch der Pächter durch." 51
\end{abstract}

In Auseinandersetzung mit dem Besitzbegriffs Jherings versucht Goudeket diesen Einwand abzuwehren. Goudeket gesteht ein, dass nach außen erkennbare Tatsachen in der Tat niemals verdeutlichen, ob Detention oder Besitz vorliegt, meint aber, dass dies auch nicht erforderlich ist, weil nur die Tatsachen in dem Moment entscheidend sind, in dem die tatsächliche Herrschaft erworben wird:

\footnotetext{
"Wanneer ik mijne boeken maar eenmaal in mijne woning ontvangen en in mijn bibliotheek geplaatst heb, dan blijf ik bezitter van die boeken, al zie ik er ook jarenlang niet naar om. (...) En wanneer dan kan worden aangetoond, dat ik de boeken in bruikleen of huur heb aangenomen, dan heb ik ook gehandeld als iemand die houder is voor een ander en zal ik ook houder blijven, zoolang ik niet bewijzen kan daden te hebben verricht, waardoor ik mij die boeken heb toegeëigend. Toch moet worden erkend dat de moeilijkheid hiermede nog niet geheel is opgelost. Immers deze beschouwing voert tot de consequentie dat de bezitter steeds zal moeten aantoonen, op welke wijze hij het bezit verkregen heeft. En dit bewijs - men zal het Ihering moeten toegeven - is dikwijls hoogst moeielijk te leveren.
}

50 Eine derartige Rückwirkung des animus befürwortet Hofmann, Het Nederlandsch Zakenrecht, 3. Auflage (1944), 67.

51 Jhering (oben Fußnote 3), 178 f. 


\section{E.F. VERHEUL}

(...). Toch is ook dit bezwaar gemakkelijk te verhelpen. De ervaring leert, dat wij van verreweg de meeste zaken, die wij in ons bezit hebben, burgerlijke bezitters zijn en dat die zaken, waarvan wij slechts houders zijn voor een ander, de minderheid uitmaken. Wij mogen daarom aannemen, dat het normale, regelmatige het burgerlijke bezit is, en dat detentie de uitzondering vormt.. ${ }^{52}$

Aus dem Zitat geht hervor, dass Goudeket sich hier unbewusst in Richtung des Jheringschen Besitzbegriffs bewegt: ${ }^{53}$ Jede tatsächliche Herrschaft über eine Sache qualifiziert sich im Ausgangspunkt als Besitz, sofern nicht wegen der Existenz eines Rechtsverhältnisses Detention vorliegt. ${ }^{54}$ Wenn man nicht auf einen rein psychologischen Willen abstellen will, ist ein solcher Ansatz auch nahezu unverzichtbar, weil auch die Tatsachen in dem Moment, in dem die tatsächliche Herrschaft erworben wird, fast nie richtungweisend sind. Wie soll sich aus der Entgegennahme eines Buches schließen lassen, ob jemand Besitzer oder Detentor ist? Dies lässt sich nur mithilfe des der Entgegennahme zugrunde liegenden Rechtsverhältnisses feststellen.

Gänzlich unhaltbar wird die animus-Lehre beim mittelbaren Besitz. Wie sollen die nach außen erkennbaren Tatsachen darauf hinweisen, dass der mittelbare Besitzer, zum Beispiel der Vermieter, die Sache innehat und dass er sie für sich selbst, also wie ein Eigentümer, innehat? Gerade der Mieter hat die tatsächliche Herrschaft über die Sache. Wenn sich der Besitz in der Tat aus zwei Elementen zusammensetzt, und zwar aus der tatsächlichen Herrschaft und dem Willen, die Sache für sich selbst innezuhaben, müsste man die tatsächliche Herrschaft des Mieters auf irgendeine Weise dem Vermieter zurechnen, sodass diese, ihm zugerechnete tatsächliche Herrschaft in Verbindung mit dem Besitzwillen dazu führen würde, dass der Vermieter als mittelbarer Besitzer die Sache in der Tat besitzt. Es ist nicht haltbar, dass die nach außen erkennbaren Tatsachen oder eben die Verkehrsauffassung darauf hindeuten, dass gerade der Vermieter die Sache besitzt. ${ }^{55}$ In Wirklichkeit ist der Vermieter nur deswegen Besitzer, weil das zwischen ihm und dem Mieter bestehende Rechtsverhältnis dazu führt, dass der Mieter nur Detentor ist und der Vermieter deshalb Besitzer bleibt. Es ist also das Rechtsverhältnis, das zu einer Negation des Besitzes in der Person des Inhabers führt und gleichzeitig ermöglicht, dass sich der Besitz kraft des Rechtsverhältnisses auf den mittelbaren Besitzer erstreckt. Der mittelbare Besitzer ist also nicht Besitzer, weil er tatsächliche Herrschaft über die Sache hat - die übt

52 Opzoomer-Goudeket, Het Burgerlijk Wetboek verklaard. Derde Deel. 3. Auflage (1911), $285 \mathrm{f}$.

53 Siehe z. B. exemplarisch Opzoomer-Goudeket (oben Fußnote 52), 289, wo angemerkt wird, dass auch dann Detention vorliegt, wenn der Inhaber nicht den Willen äußert, die Sache für einen anderen innezuhaben, sondern dessen ungeachtet in einem Rechtsverhältnis steht, aufgrund dessen er die Sache innehat, sodass man den Detentionswillen nicht wirklich aufzuspüren braucht.

54 In diese Richtung geht auch die Argumentation Hofmanns, der Jhering und Scholten zwar stark kritisiert, im Ergebnis jedoch zu vergleichbaren Ergebnissen gelangt, indem er anmerkt, dass der Wille des Detentors dadurch objektiviert werden muss, dass vom Normallfall auszugehen ist. Siehe Hofmann (oben Fußnote 50), 66. Durch eine solche Objektivierung wird in Wirklichkeit auf den üblichen Inhalt des Rechtsverhältnisses und damit auf das Rechtsverhältnis als solches abgestellt.

55 Van Oven, WPNR 4539 (1958), 241, 242. 


\section{DIE REZEPTION DES BESITZBEGRIFFS JHERINGS}

gerade der Inhaber, und nicht der mittelbare Besitzer aus - sondern nur, weil das Rechtsverhältnis ihm den Besitz vermittelt.

\subsection{Das Rechtsverhältnis als entscheidender Anknüpfungspunkt}

Richtet man den Blick nicht nur auf die Besitzdefinition von Artikel 3:107 BW und das Kriterium der Verkehrsauffassung aus Artikel 3:108 BW, sondern betrachtet man den ganzen Abschnitt des Besitzrechts, zeigt sich ganz deutlich, dass der niederländische Gesetzgeber dem Besitzrecht den bereits unter Geltung des alten niederländischen Gesetzbuchs rezipierten Besitzbegriff Jherings zugrunde gelegt hat. ${ }^{56}$ Der weitgreifende Einfluss Jherings tritt vor allem in den gesetzlichen Bestimmungen, die auf Artikel 3:108 BW folgen, in den Vordergrund. Aufgrund des Artikels 3:108 BW muss zwar anhand der Verkehrsauffassung beurteilt werden, ob Besitz oder Detention vorliegt, jedoch "unter Berücksichtigung der nachstehenden Bestimmungen". Wenn diese gesetzlichen Bestimmungen zu einem abweichenden Ergebnis führen, haben die gesetzlichen Bestimmungen Vorrang. ${ }^{57}$ Ins Auge fällt, dass einige dieser Bestimmungen gerade dazu dienen, Detention in Situationen eintreten zu lassen, in denen Detention aufgrund der Verkehrsauffassung nicht (ohne Weiteres) eintreten würde. Damit wird beabsichtigt, jemandem in einer bestimmten Situation auch dann Detention zuzusprechen, wenn aufgrund der Verkehrsauffassung Besitz entstehen würde, sodass sich die mit dem Besitz verbundenen Rechtswirkungen auf einen anderen erstrecken können. ${ }^{58}$

Mit anderen Worten: Aufgrund dieser gesetzlichen Bestimmungen tritt bisweilen bei demjenigen, der die tatsächliche Herrschaft ausübt, nur Detention ein, um einem anderen, der die tatsächliche Herrschaft gerade nicht ausübt, die Rechtsstellung des Besitzers einzuräumen. In diesem Zusammenhang merken Scholten und Beekhuis an, dass der Besitz im Ausgangspunkt ein soziologischer Begriff ist, dieser Begriff aber juristischer Natur wird, weil das Recht häufig von der Verkehrsauffassung und nach außen erkennbaren Tatsachen abweichende Rechtsfolgen eintreten lässt. ${ }^{59}$ Die Qualifikation einer tatsächlichen Herrschaft als Detention ist eine rechtliche Konstruktion, die dazu dient, denjenigen, der - mangels einer solchen rechtlichen Konstruktion - normalerweise den Besitz innehaben würde (weil er die tatsächliche Herrschaft ausübt), zum Detentor werden zu lassen, sodass die Position

56 So ist auch der Befund Van Ovens, WPNR 4539 (1958), 241 ff. und Mijnssens, WPNR 5165 (1972), $125,126$.

57 Bartels, De titel van overdracht in driepartijenverhoudingen (2004), 41, Rank-Berenschot (oben Fußnote 44), 23, Jansen, RMThemis 174 (2013), 2, 3 und Van Schaick, Rechtsgevolgen en functies van bezit en houderschap, 2. Auflage (2014), 17.

$58 \quad$ Van Oven, WPNR 4539 (1958), 241, 242.

59 Asser-Scholten (oben Fußnote 13), 43 und Beekhuis, WPNR 4460 (1956), 393. 


\section{E.F. VERHEUL}

des Besitzers einem anderen zugesprochen werden kann. ${ }^{60}$ Aus der Ausübung der tatsächlichen Herrschaft durch den Detentor ergibt sich diese Einschränkung seiner Stellung in aller Regel nicht: Aus der Art und Weise, in der der Detentor mit der Sache umgeht, ist nicht abzulesen, ob er ein besseres Recht eines anderen respektiert. ${ }^{61}$ Dies ergibt sich nur aus dem Rechtsverhältnis zwischen Detentor und Besitzer, das für die Außenwelt weder wahrnehmbar noch erkennbar ist. Nur anhand des Rechtsverhältnisses lässt sich beurteilen, ob Besitz oder Detention vorliegt. ${ }^{62}$ Die Existenz eines solchen Rechtsverhältnisses hat zur Folge, dass in der Person des Inhabers Detention eintritt und demjenigen, der nicht die tatsächliche Herrschaft über die Sache hat, gerade der Besitz zugesprochen wird.

Diese Struktur des niederländischen Besitzrechts führt also im Ergebnis dazu, dass aufgrund der Grundregel des Artikels 3:108 BW normalerweise Besitz vorliegt, wenn jemand die tatsächliche Herrschaft über die Sache ausübt, und nur ausnahmsweise aufgrund der nachfolgenden gesetzlichen Bestimmungen Detention eintritt. Entscheidend ist aufgrund dieser gesetzlichen Bestimmungen (Art. 3:110 und 3:111 BW), ob zwischen zwei Personen ein Rechtsverhältnis gegeben ist, nach dessen Inhalt die eine Person eine Sache für die andere Person innehat. Anhand des Rechtsverhältnisses muss also beurteilt werden, ob jemand eine Sache für einen anderen innehat. Für die Frage, ob jemand die Sache für sich selbst oder für einen anderen innehat, ist letztendlich also das Rechtsverhältnis maßgeblich, weil die gesetzlichen Bestimmungen dem Rechtsverhältnis entscheidende Bedeutung beimessen und diese gesetzlichen Bestimmungen vor dem Verkehrsauffassungskriterium des Artikels 3:108 BW Vorrang haben. Liegt kein solches Rechtsverhältnis vor, ist die tatsächliche Herrschaft über eine Sache ohne Weiteres als Besitz zu deuten. ${ }^{63}$ Im Ergebnis führt diese Systematik also tatsächlich zum Besitzbegriff Jherings, wonach Besitz aufgrund des Kriteriums der Verkehrsauffassung der gegebene Ausgangspunkt ist, während Detention nur dann eintritt, wenn ein Rechtsverhältnis gegeben ist.

Beispielhaft für diese Systematik ist auch die jüngere Rechtsprechung des Hoge Raad über das (ursprünglich aus dem französischen Recht übernommene) Erfordernis der Unzweideutigkeit (ondubbelzinnigheid) des Besitzes. ${ }^{64}$ Dieses Erfordernis konnte einen Besitzerwerb verhindern, wenn die tatsächliche Herrschaft nicht unzweideutig auf Besitz hindeutete. Im Jahre 2015 hat der Hoge Raad die Bedeutung dieses Erfordernisses jedoch stark zurückgedrängt. Der Umstand, dass bestimmte Handlungen auch von einem Detentor vorgenommen

60 Siehe Van Schaick (oben Fußnote 57), 17, der anmerkt, dass die Situation dergestalt sein kann, dass man aufgrund der nach außen erkennbaren Tatsachen auf Besitz schließen würde, jedoch aufgrund gesetzlicher Anordnung Detention eintritt.

61 Vgl. Suijling (oben Fußnote 39), 107: Jeder Inhaber einer Sache macht nach außen den Eindruck, die Sache für sich selbst innezuhaben.

62 Vgl. auch, wenngleich am animus-Erfordernis festgehalten wird, Rank-Berenschot (oben Fußnote 44), 18: "Normalerweise ist die Existenz eines Rechtsverhältnisses eine negative Abgrenzung."

63 Für das Eintreten von Detention ist die Existenz eines Rechtsverhältnisses also erforderlich. Siehe AsserBartels/Van Mierlo, Algemeen goederenrecht, 16. Auflage (2013), 105 und Van Schaick (oben Fußnote 57), 9. A.A. Snijders/Rank-Berenschot (oben Fußnote 44), 98. 


\section{DIE REZEPTION DES BESITZBEGRIFFS JHERINGS}

werden können, steht einem Besitzerwerb nicht entgegen, sofern nicht tatsächlich Anknüpfungspunkte vorhanden sind, die auf Detention hindeuten. Diese Rechtsprechung qualifiziert die Ausübung der tatsächlichen Herrschaft also normalerweise als Besitz. Auch daraus ist ersichtlich, dass dem Willen keine Bedeutung beigemessen wird: Selbst wenn die nach außen erkennbaren Tatsachen nicht ohne Weiteres auf einen Besitzwillen hindeuten, tritt dennoch Besitz ein, wenn keine Anhaltspunkte für die Existenz eines Rechtsverhältnisses vorliegen.

Die entscheidende Bedeutung des Rechtsverhältnisses als Abgrenzungskriterium zwischen Besitz und Detention tritt ganz prägnant in Artikel 3:110 BW hervor: ${ }^{65}$ Ist zwischen zwei Personen ein Rechtsverhältnis gegeben, nach dessen Inhalt die eine Person eine Sache für die andere Person innehat, so hat die eine Person die Sache auch tatsächlich für die andere Person inne, sobald sie die Sache zur Ausführung des Rechtsverhältnisses erhält. Nicht der Wille desjenigen, der die tatsächliche Herrschaft ausübt, sondern das Rechtsverhältnis, vermöge dessen er die Sache für einen anderen innehat, ist also maßgeblich dafür, dass nicht Besitz, sondern Detention vorliegt. ${ }^{66}$ Dies führt zum Beispiel dazu, dass derjenige, der eine Sache kauft - trotz der nach außen erkennbaren Tatsachen nur Detentor wird, wenn er die Sache im Auftrag eines anderen erwirbt, selbst wenn die Existenz des zugrunde liegenden Rechtsverhältnisses auf keinerlei Weise nach außen hervortritt und sogar auch dann, wenn derjenige, der die Sache tatsächlich empfängt, in dem Moment einen anderslautenden Willen an den Tag legt. ${ }^{67}$ Der Artikel bildet damit die Grundlage für den Direkterwerb im Fall der mittelbaren Stellvertretung: Weil zwischen mittelbarem Stellvertreter und Geschäftsherrn ein Rechtsverhältnis existiert, vermöge dessen der mittelbare Stellvertreter die Sache für den Geschäftsherrn erwerben wird, erwirbt der mittelbare Stellvertreter die zur Ausführung dieses Rechtsverhältnisses erworbene Sache auch ohne Weiteres für den Geschäftsherrn.

Nun könnte man anscheinend einwenden, dass der Wille in diesem Zusammenhang durchaus Bedeutung hat, da die bloße Existenz des Rechtsverhältnisses für das Eintreten der Detention nicht ausreicht, weil notwendig ist, dass die Sache auch "zur Ausführung des Rechtsverhältnisses" erworben wird. Diese Argumentation ist jedoch verfehlt. Ob die Sache zur Ausführung des Rechtsverhältnisses erworben wird, kann nur anhand des Rechtsverhältnisses selbst beurteilt werden, da die Bedingungen und Voraussetzungen des Rechtsverhältnisses ergeben, ob die Sache zur Ausführung dieses Rechtsverhältnisses erworben wird. So tritt auch dann Detention ein, wenn der Stellvertreter die vom Geschäftsherrn begehrte Sache kauft und sich dabei von den Rahmenbedingungen des Rechtsverhältnisses leiten lässt, zum Beispiel indem er den Höchstpreis berücksichtigt und die Sache mit den

65 Vgl. Asser-Bartels/Van Mierlo (oben Fußnote 63), 105.

66 Pitlo-Reehuis/Heisterkamp (oben Fußnote 47), 304. Siehe auch die Materialien zum BW (Van Zeben/Du Pon (oben Fußnote 44), 430): "nicht ein subjektiver Wille des Inhabers im Moment des Erwerbs entscheidet, sondern das zwischen den Parteien existierende Rechtsverhältnis."

67 Vgl. Langemeijer, NJB 38 (1963), 666, 669. Siehe auch die Materialien zum BW (Van Zeben/Du Pon (oben Fußnote 44), 430): "Der Beweis eines anderslautenden Willens ist nicht relevant." 


\section{E.F. VERHEUL}

vom Geschäftsherrn zur Verfügung gestellten Geldern erwirbt, jedoch in der tatsächlichen Ausführung einen anderslautenden Willen äußert. Kauft der Stellvertreter hingegen eine völlig andere Sache, erwirbt er sie selbstverständlich nicht für den Geschäftsherrn, aber nicht, weil sein Wille dazu führt, sondern lediglich deshalb, weil dieser Erwerb außerhalb des Geltungsbereiches des Rechtsverhältnisses liegt. Das Rechtsverhältnis ist also entscheidend, da geprüft werden muss, ob der Erwerb innerhalb des Geltungsbereiches des Rechtsverhältnisses liegt. ${ }^{68}$

Eine ähnliche Bindung an das Rechtsverhältnis schafft Artikel 3:111 BW. Wenn jemand angefangen hat, eine Sache kraft eines Rechtsverhältnisses für einen anderen innezuhaben, so dauert diese Detention so lange fort, wie sich daran nichts ändert, und zwar entweder infolge einer Handlung desjenigen, für den der Detentor die Sache innehat, oder infolge eines Widerspruchs seines Rechts (nemo causam possessionis sibi ipse mutare potest). Auch in diesem Fall legt das Rechtsverhältnis die Detention auf zwingende Weise fest und schiebt den Willen desjenigen, der die Sache innehat, und die nach außen erkennbaren Tatsachen beiseite: Auch wenn der Detentor seinen Besitzwillen in die Tat umsetzt, indem zum Beispiel der Mieter eines Fahrrads den Fahrradreifen wechselt oder der Mieter eines Hauses einen Heizkessel einbaut, bleibt er aufgrund des Rechtsverhältnisses lediglich Detentor. ${ }^{69}$ Erforderlich für einen solchen Widerspruch des Rechts ist, dass der Detentor dem Recht desjenigen, für den er die Sache innehat, ihm gegenüber widerspricht. Der Widerspruch muss also dem mittelbaren Besitzer gegenüber erfolgen. Dies hat zur Folge, dass das bloße Abschließen eines Rechtsverhältnisses mit einem Dritten, kraft dessen der Inhaber die Sache für den Dritten innehaben wird (Besitzkonstitut), nicht zum Besitzverlust des mittelbaren Besitzers führt, da der Inhaber aufgrund des früheren Rechtsverhältnisses die Sache bereits für den mittelbaren Besitzer innehat und sich nur durch einen Widerspruch dessen Rechts von diesem Rechtsverhältnis loslösen kann, sodass der Dritte den Besitz nicht erwerben kann, solange ein derartiger Widerspruch nicht stattgefunden hat. ${ }^{70}$

68 A.A. Beekhuis, WPNR 4461 (1956), 405, 406, weil das Entstehen eines Rechtsverhältnisses vom Willen abhängig ist. Selbstverständlich ist für das Zustandekommen des Rechtsverhältnisses Willensübereinstimmung erforderlich, sodass der Wille in dem Zusammenhang eine Rolle spielt. Es handelt sich jedoch gerade um die Frage ob, wenn ein solches Rechtsverhältnis vorliegt, das Rechtsverhältnis oder ein entgegengesetzter Wille entscheidend ist. Wenn ein derartiges Rechtsverhältnis vorliegt, hat ein gegensätzlicher Wille keinen Einfluss, sodass im Ergebnis das Rechtsverhältnis entscheidend ist. Vgl. AsserScholten (oben Fußnote 13), 51 und Mijnssen, WPNR 5165 (1972), 125, 126. Siehe auch die Materialien zum BW (Van Zeben/Du Pon (oben Fußnote 44), 430): "Der Beweis eines anderslautenden Willens ist nicht relevant. Erst wenn die in [Artikel 3:111 BW] genannten Tatbestände vorliegen, ist das Rechtsverhältnis nicht mehr maßgeblich." Gegen Beekhuis auch Van Oven, WPNR 4539 (1958), 241, 242.

69 Von Snijders/Rank-Berenschot (oben Fußnote 44), 99 wird diese Regel hingegen gerade als eine Ausnahme auf die normalerweise durchaus entscheidende Rolle des Willens angesehen. Damit wird jedoch verkannt, dass auch die gesetzlichen Bestimmungen, insbesondere Artikel 3:111 BW, den Inhalt des Besitzbegriffs bestimmen. Siehe dazu die Materialien zum BW (Van Zeben/Du Pon (oben Fußnote 44), 425), in denen angemerkt wird, dass es eben auch die gesetzlichen Bestimmungen sind, die den Inhalt des Besitzbegriffs näher festlegen.

70 Dazu GS Vermogensrecht-Verheul (2018), art. 3:90 BW, Rn. 1.12.2.2 m.w.N. 


\section{DIE REZEPTION DES BESITZBEGRIFFS JHERINGS}

In der älteren Literatur wurde von einigen Autoren versucht, diese Bindung des Detentors mit der animus-Lehre zu versöhnen, indem von einer qualifizierten Äußerung eines anderslautenden Besitzwillens gesprochen wurde, sodass im Ergebnis immer noch der Wille entscheiden sei. ${ }^{71}$ Diese Ansicht verkennt jedoch, dass auch in dieser Situation nur anhand der Beziehung zwischen mittelbarem Besitzer und Detentor entschieden werden kann, ob Detention oder Besitz vorliegt. Selbstverständlich ist für einen Widerspruch des Rechts des mittelbaren Besitzers eine Äußerung eines derartigen Widerspruchs erforderlich. Daraus lässt sich allerdings nicht schließen, dass letztendlich der nach außen erkennbare Wille entscheidend sei. Nur aufgrund der Kenntnis der Beziehung zwischen Detentor und mittelbarem Besitzer und der Kenntnis dessen, was sich in dieser Beziehung ereignet hat, lässt sich feststellen, ob Detention oder Besitz vorliegt. Auch hier hängt die Frage, ob Detention oder Besitz vorliegt, letztendlich von der Beziehung zwischen dem (früheren) mittelbaren Besitzer und dem Detentor ab.

Schließlich ermöglicht die den Artikeln 3:110 und 3:111 BW zugrunde liegende Technik, bei der das Rechtsverhältnis darüber entscheidet, ob Detention oder Besitz vorliegt, auch die Bindung bei der antizipierten Übereignung. ${ }^{72}$ Wird eine zukünftige Sache antizipiert übereignet, so ist bedeutungslos, ob der Veräußerer die Sache in dem Moment, in dem er sie tatsächlich erwirbt, auch noch wirklich für den Erwerber innehaben will: Das Rechtsverhältnis zwischen Veräußerer und Erwerber führt dazu, dass der Veräußerer die Sache ohne Weiteres für den Erwerber innehat, sodass Letzterer in dem Moment auch automatisch das Eigentum erwirbt.

\section{Die Subjektivitätstheorie im deutschen Recht}

Jherings Kritik der Subjektivitätstheorie war nicht nur dogmatischer, sondern vor allem rechtspolitischer Natur: Jhering hatte vor allem die Interessen des mittelbaren Besitzers vor Augen. Er hielt es für unverdaulich, dass eine von dem Recht geschützte Rechtsposition von so etwas Nichtigem und Flüchtigen wie dem Willen desjenigen abhängt, der die Sache für ihn innehat. Damit werde der Besitz zum "Spielball der Laune" des Detentors. ${ }^{73}$ Obwohl er damit in seinen Auseinandersetzungen der Besitzlehre Savignys nicht völlig gerecht wird, ${ }^{74}$

71 Opzoomer-Goudeket (oben Fußnote 52), 290 f. und Hofmann (oben Fußnote 50), 67)

72 Zutreffend ist also, dass die Bindung bei der antizipierten Übereignung in den Materialien zum BW mit der Bindung an das Rechtsverhältnis aufgrund von Artikel 3:110 BW in Verbindung gebracht wird. Siehe Reehuis/Slob, Parlementaire Geschiedenis van het Nieuwe Burgerlijk Wetboek. Invoering Boeken 3, 5 en 6. Boek 3 (1990), 1251. Siehe bereits Meijers (oben Fußnote 36), 253. Vgl. auch Schuijling, Levering en verpanding van toekomstige goederen (2016), 212.

73 Jhering (oben Fußnote 3), 15, ausf. 208 ff.

74 Gerade Savigny zufolge reicht eine bloße Willensänderung für eine Umwandlung von Detention in Besitz nicht aus. Notwendig sei, dass der Detentor sich die Sache dadurch zueigne, dass er ein furtum begeht, was eine körperliche Handlung bezüglich der Sache (sog. contrectatio) voraussetzt. Siehe Savigny (oben Fußnote 1), 365 ff. Ihm folgend z.B. Sintenis (oben Fußnote 2), 460 ff. a. A. Brinz (oben 


\section{E.F. VERHEUL}

fällt ins Auge, dass im heutigen deutschen Recht die von Jhering genannten Bedenken hingegen durchaus auftauchen, ${ }^{75}$ indem der mittelbare Besitz völlig von dem Besitzmittlungswillen des Besitzmittlers abhängig ist.

Gibt der Besitzmittler seinen Besitzwillen dadurch auf, dass er einen anderslautenden Willen kundgibt oder mit einem Dritten ein Rechtsverhältnis abschließt, vermöge dessen er den Besitz fortan für den Dritten mitteln wird, so verliert der mittelbare Besitzer seinen Besitz, sogar auch dann, wenn er von der Aufgabe des Besitzmittlungswillens nichts erfährt. ${ }^{76}$ Erforderlich ist nur, dass die Aufgabe des Besitzmittlungswillens auf irgendeine Weise nach außen hervortritt. Dies ist zum Beispiel der Fall, wenn der Besitzmittler seinen Namen in ein geliehenes Buch schreibt, ein Rechtsverhältnis mit einem Dritten schließt oder wenn er schlichtweg einem Dritten gegenüber erklärt, die Sache fortan für sich selbst innezuhaben. ${ }^{77}$ Jhering betrachtete dies als die äußerste Konsequenz der Subjektivitätstheorie:

\footnotetext{
"Die äußerste Consequenz, die freilich angesichts der Quellen Niemand gezogen hat und ziehen konnte, würde darin bestehen, daß die Art des Besitzverhältnisses gänzlich der Willensbestemmung des Inhabers überlassen sei, daß er, mithin, da er sich selber durch seinen Willen nicht binden kann, seinen Entschluß beliebig ändern, heute detiniren, morgen besitzen, dann wieder detiniren könne, und so weiter fort, - das Besitzverhältnis ein reiner Spielball der Laune der Parteien, an Stelle der objectiven Rechtsregel der individuelle Wille der Partei, an Stelle der Rechtssicherheit die Rechtsunsicherheit, an Stelle der Ordnung die Anarchie!" 78
}

Diese Interpretation des Erfordernisses eines Besitzmittlungswillens als notwendige Voraussetzung für den Fortbestand des mittelbaren Besitzes hat auch weitreichende praktische Konsequenzen. Allbekannt sind die Fälle, in denen der Besitzmittler mit einem Dritten ein Rechtsverhältnis vereinbart, vermöge dessen er den Besitz für den Dritten mittelt und wodurch der erste mittelbare Besitzer seinen Besitz verliert. ${ }^{79}$ So kann der Vorbehaltskäufer den mittelbaren Besitz des Vorbehaltsverkäufers dadurch zerstören, dass er mit einem Dritten (durchaus eine Bank) ein Besitzmittlungsverhältnis schließt. Entschließt der Vorbehaltskäufer sich später, den Besitz wieder für den Vorbehaltsverkäufer zu mitteln, hat dies zur

Fußnote 2), 528 ff., Randa (oben Fußnote 1), 512 f. und Windscheid-Kipp (oben Fußnote 1), 808. Zum Streitstand ausführlich Ernst, Eigenbesitz und Mobiliarerwerb (1992), 228 ff.

75 Zutr. Ernst (oben Fußnote 74), 229, der anmerkt, dass die heutige deutsche Lehre die von Jhering bekämpfte Subjektivitätstheorie verwirklicht.

76 Wieling, Sachenrecht, 2. Auflage (2006), 245, Baur/Stürner, Sachenrecht, 18. Auflage (2009), 80, Westermann/Gursky/Eickmann, Sachenrecht, 8. Auflage (2011), 137, Staudinger/Gutzeit (2012), § 868 BGB, Rn. 86, MünchKomm-BGB/Joost (2017), § 868 BGB, Rn. 30. Aus der Rechtsprechung z.B. BGH, Urt. v. 10.11.2004, NJW 2005, 359.

77 Westermann/Gursky/Eickmann (oben Fußnote 76), 137 und Wilhelm, Sachenrecht, 5. Auflage (2016), 249.

78 Jhering (oben Fußnote 3), $14 \mathrm{f}$.

79 Es handelt sich hier um die sogenannten Zucker- und Fräsmaschinen-Fälle: RG, Urt. v. 05.02.1932, RGZ 135, 75, RG, Urt. v. 11.11.1932, RGZ 138, 265 und BGH, Urt. v. 27.03.1968, NJW 1968, 1382. 


\section{DIE REZEPTION DES BESITZBEGRIFFS JHERINGS}

Folge, dass der Vorbehaltsverkäufer erneut Besitzer wird und der Dritte seinen Besitz verliert. ${ }^{80}$ Die von Jhering befürchtete Möglichkeit, dass der Detentor sich durch eine Willensänderung immer wieder für Detention für den einen, Detention für den anderen und sogar Besitz entscheiden könnte, ${ }^{81}$ hat sich damit für das deutsche Recht bewahrheitet. Sie hat damit außerdem nicht nur Konsequenzen für den Besitz des ersten mittelbaren Besitzers, sondern kann über die Regeln des Erwerbs vom Nichtberechtigten überdies zum Eigentumsverlust führen. ${ }^{82}$

Aus niederländischer Sicht fällt ins Auge, dass gerade diese logischen Konsequenzen der Subjektivitätstheorie in der deutschen Literatur durchaus als unangemessen empfunden werden. Sie bemüht sich daher auch, diese Konsequenzen zu mildern, entweder dadurch, dass der mittelbare Besitzer seinen Besitz behält oder dadurch, dass der mittelbare Besitzer nicht über den Besitzverlust hinaus auch das Eigentum verliert. ${ }^{83}$

Ein nicht unbeträchtlicher Teil der deutschen Lehre meint, dass das Abschließen eines neuen Besitzmittlungsverhältnisses zwar zum Besitzerwerb des Dritten führe, dieser Besitz jedoch geringerer Qualität sei, weil es nur zu einem durch den bereits existierenden mittelbaren Besitz beschränkten Nebenbesitz führe: Der Besitzmittler mittelt den Besitz folglich gleichzeitig den beiden mittelbaren Besitzern. Weil dieser Nebenbesitz geringerer Qualität sei, genüge er nicht zu einem gutgläubigen Erwerb. ${ }^{84}$ Faktisch nimmt diese Lehre also an, dass der Erwerber den Besitz nicht wirklich erwirbt. ${ }^{85}$ $\mathrm{Zu}$ vergleichbaren Ergebnissen führt die sogenannte Identitätstheorie, die den mittelbaren Besitz dem Rechtsverhältnis gleichsetzt. ${ }^{86}$ Nach diesem Ansatz geht der mittelbare Besitz erst dann verloren, wenn der Besitzmittler das Rechtsverhältnis beendet, kraft dessen er den Besitz für den anderen mittelt, was nur dem mittelbaren Besitz gegenüber geschehen kann.

Nach einem dritten Ansatz kann der mittelbare Besitz zwar durch eine Änderung des Besitzmittlungswillens verloren gehen, der Besitzerwerb eines Dritten kann aber niemals zum Eigentumserwerb führen, solange der Besitzmittler die Sache noch innehat. Der ältere Herausgabeanspruch des ursprünglichen mittelbaren Besitzers blockiert nach dieser Auffassung einen Gutglaubenserwerb. ${ }^{87}$

Das niederländische Recht verwirklicht diese Bestrebungen dadurch, dass es die Detention nicht vom Willen des Detentors abhängen, sondern so lange fortdauern lässt, bis der Detentor dem Recht des Besitzers ihm gegenüber widerspricht. Damit verwirklicht das niederlän-

\footnotetext{
80 So ausdrücklich MünchKomm-BGB/Joost (2017), § 868 BGB, Rn. 20.

81 z. B. Jhering (oben Fußnote 3), 229 f.

82 Siehe dazu die in Fußnote 79 genannte Rechtsprechung.

83 Einen Überblick bietet MünchKomm-BGB/Oechsler (2017), § 934 BGB, Rn. 6-8.

84 Medicus, Gedanken zum Nebenbesitz, FS Hübner (1984), 611ff., Wieling (oben Fußnote 76), 246, Baur/ Stürner (oben Fußnote 76), 672 und scheinbar auch Staudinger/Gutzeit (2012), § 868 BGB, Rn. 9.

85 Ganz prägnant Medicus (oben Fußnote 84): "Die Funktion des Nebenbesitzes ist also ganz negativ."

86 Boehmer, Grundlagen der bürgerlichen Rechtsordnung. II/2 (1952), 41 ff. Siehe für weitere Nachweise Wieling (oben Fußnote 76), 245, Fn. 53.

87 Picker, AcP 188 (1988), 511, 569 und Hager, Verkehrsschutz durch redlichen Erwerb (1990), 362 ff.
} 


\section{E.F. VERHEUL}

dische Recht die Ziele Jherings: Es lässt den Willen des Detentors außer Betracht, entscheidet zwischen Detention und Besitz anhand des Rechtsverhältnisses und bewirkt damit, dass der mittelbare Besitzer seinen Besitz nicht ohne Bekanntgabe durch den Detentor verliert. ${ }^{88}$

\section{Die Bilanz}

Will man Bilanz ziehen und eine Entscheidung zugunsten der Subjektivitätstheorie oder des Besitzbegriffs Jherings treffen, muss man durchschauen, dass beide Theorien unterschiedliche Ziele verfolgen. In der Subjektivitätstheorie kann die Frage, wer Besitzer einer Sache ist, eher anhand der nach außen erkennbaren Tatsachen entschieden werden, während nach dem Besitzbegriff Jherings nicht immer ersichtlich ist, wer Besitzer einer Sache ist, weil das Rechtsverhältnis ausschlaggebend ist. Der Besitzbegriff Jherings hat hingegen zur Folge, dass der mittelbare Besitzer seinen Besitz nicht ohne sein Wissen verliert.

Die Entscheidung zugunsten der einen oder der anderen Theorie wird vor allem von diesen rechtspolitischen Fragen abhängen. Will man strikt am tatsächlichen Charakter des Besitzes festhalten, wird die Entscheidung eher zugunsten der Subjektivitätstheorie ausfallen, weil sich mit dem tatsächlichen Charakter des Besitzes nicht vertragen würde, dass der Detentor auch dann Detentor bleibt, wenn er sich nach außen wie ein Eigentümer verhält und dies nur dem Besitzer gegenüber nicht kundgegeben hat. ${ }^{89}$ Zugleich muss man bei diesem Ansatz in Kauf nehmen, dass der mittelbare Besitzer seinen Besitz auch schon verlieren kann, wenn er davon nichts erfährt. ${ }^{90}$

Emil Verheul ${ }^{91}$

Groningen

\footnotetext{
88 Vgl. Suijling (oben Fußnote 39), 114 und Van Oven, WPNR 4539 (1958), 241, 242.

89 Statt vieler Ernst (oben Fußnote 74), 233f., Wieling (oben Fußnote 76), 245 und Wilhelm (oben Fußnote 77), 247.

90 Eine mittlere Position, die gute historische Zeugnisse vorweisen kann (dazu oben Fußnote 74), haben Ernst (oben Fußnote 74), 233 ff. und Knütel, FS Lange (1992), 903, 924 f. eingenommen: Die Beendigung des mittelbaren Besitzers setze eine sachbezogene Handlung des Besitzmittlers voraus, wodurch er sich dem mittelbaren Besitzer gegenüber als Vindikationsbesitzer haftbar mache.

91 Dr. E.F. Verheul ist Dozent Privatrecht an der Universität Groningen.
} 